\title{
FACTORS INFLUENCING DOCUMENTATION IN NURSING CARE BY NURSES AT THE TAMALE TEACHING HOSPITAL, GHANA
}

\author{
Seidu ${ }^{1}$, A. A., Abdulai ${ }^{1}$, A., Aninanya ${ }^{2 *}$, G. A. \\ ${ }^{1}$ School of Nursing and Midwifery, University for Development Studies, Tamale, Ghana. \\ ${ }^{2}$ Department of Health Services Policy, Planning, Management and Economics, School of Public Health, \\ University for Development Studies, Tamale, Ghana. \\ *Corresponding Author's Email: ganinanya@uds.edu.gh
}

\begin{abstract}
In Ghana, nursing documentation practice by nurses is sub-optimal. This analytical cross-sectional study assessed sociodemographic determinants of nursing documentation practice among 278 nurses at the Tamale Teaching Hospital (TTH). Data was gathered using a questionnaire and both descriptive and inferential analyses were done to determine factors influencing nursing documentation practice. A large majority of the respondents $(84.6 \%)$ had adequate knowledge on nursing documentation. Most of them (84.2\%) had positive attitudes towards it and a large majority of them (77.1\%) asserted to practice it always but only $74.0 \%$ adequately practiced nursing documentation. Knowledge of nursing documentation was statistically associated with age (AOR 0.12, 95\% C1: 0.029-1.507; $p=0.004$ ) and work experience (AOR 15.29, 95\% C1: 3.083-75.872; $p=0.001$ ), males were significantly more likely to have positive attitudes towards it (AOR, $2.81 \mathrm{CI}, 1.434-5.501, p=0.003)$ whilst respondents aged 21-30 years (AOR, 5.85 (2.64-12.97), $p<0.001)$ and certificate nurses (AOR, 23.79 (2.23-253.25), $p=0.009)$ were shown to have significantly adequate level of practice of nursing documentation. Also, knowledge of nursing documentation had a significant influence on practice of nursing documentation (AOR 0.137, 95\% C1: 0.032-0.583, $p=0.007$ ). Organizational factors that affected documentation included 'availability of operational standards', 'in-service training', lack of staff', 'lack of motivational packages and apathy'. Knowledge, attitude and practice of nurses on documentation was high but certain organisational factors could influence this. To strengthen documentation by nurses, it is recommended that nursing managers and policymakers equip TTH with nursing staff, logistics and guidelines on nursing documentation.
\end{abstract}

Keywords: Nurses, Documentation, Knowledge, Attitude, Practice, Tamale Teaching Hospital.

\section{Introduction}

Globally, nursing documentation is very important in the health system (Asamani et al., 2015). Nurses have direct contact with clients and are obliged to keep comprehensive records of all care rendered to these clients for stakeholders to be well informed in the health system (Mbabazi \& Cassimjee, 2006; McBride et al., 2012). Documentation provides a comprehensive account of care provided to patients and it serves as a legal record that specifies the care and the progress of the patient (Keenan et al., 2008; Machudo \& Mohidin, 2015). Good nursing practice demands that the care is comprehensively, timely and accurately kept on record (Mutshatshi et al., 2018b). It is imperative that the nurse makes the documentation clear, succinct and legible in a legally cautious manner to reduce the chances of misinterpretation, legal liability and adverse patient outcomes resulting from poor communication (Buunaaisie et al., 2018).

Accurate and timely documentation reflects on the care provided, promotes collaboration and communication among the health team, meets professional and legislative standards and facilitates patient care decisions and outcomes of care (Priest et al., 2007; Blake-Mowatt et al., 2013). 
Furthermore, on a broader scope, documentation is important for education, research, quality assurance, health planning, nursing development, allocation of resources and reimbursement by third party claimants ( DeLaune et al, 2003; Urquhart et al., 2018). A study conducted in Nigeria showed that documentation ensures continuity of care, allows for early detection of problems, and ensure high standard of clinical care (Hussainat, 2015). Despite the importance of documentation, nursing reports are frequently incomplete and of poor quality which compromises patient care and undermines the credibility of the nurse (Porter et al., 2004; Broderick \& Coffey, 2013; Denilsen et al., 2016; Buunaaisie et al., 2018). Improper charted vital signs imply wrong treatment plans and uncharted served medication imply probable over dose of patients which may lead to poor patient outcomes and increased healthcare cost (Okaisu et al., 2014; Obioma, 2017).

In developing countries, several factors have been reported to influence the practice of nursing documentation. A descriptive cross-sectional study that investigated factors affecting documentation practice among nurses in a hospital in Ethiopia revealed that the practice of nursing care documentation was inadequate and factors associated with it included inadequacy of documenting sheets and time (Andualem et al., 2019). In addition, Kamil et al., (2018b) asserted in a qualitative study among nurses in Indonesia that nursing documentation remains a problem and pointed out factors such as inadequate supervision on documentation, poor competency issues and lack of confidence and motivation on documentation. Again, a cross-sectional study among nurses in Ethiopia revealed poor knowledge, attitude and practice of documentation and pointed associated factors such as poor staff saturation and lack of documentation guidelines (Andualem et al., 2019). In Uganda, organizational issues, inadequate knowledge on documentation, lack of training and motivation were reported as barriers to effective nursing documentation (Nakate et al., 2015). In
South Africa, a qualitative study revealed that challenges associated with documentation include lack of time to complete records, increased patient to nurse ratio and shortage of documentation logistics (Mutshatshi et al., 2018b). Alongside these deficiencies, nurses' low knowledge and poor attitudes have been cited to impact negatively on documentation practices (Deriba et al., 2017). Challenges associated with nursing documentation could be addressed through continuous training, monitoring and evaluation of nurses (Mutshatshi et al., 2018b; Kamil et al., 2018), motivation, provision of documentation policies and materials (Nakate et al., 2015), employment of more nurses, proper time management and penalizing nurses for poorly written documents (Hussainat, 2015).

In Ghana, nursing documentation is sub-optimal and contributes to adverse health outcomes ( Okine, 2017; Buunaaisie et al., 2018). Recent developments in the Ghanaian health sector have seen health workers being served with lawsuits and other forms of disciplinary proceedings (Asamani et al., 2015). There have been reports of some cases of medical negligence and even law suits among some health professionals( Joga, 2009; Oyebode, 2013). In these cases, accurate documentation procedures would definitely have played a key role in preventing such issues. Also, despite the fact that the Nursing and Midwifery Council (NMC) regulates the training and practice of nursing and midwifery, NMC is lagging behind due to the fact that no national and/or local guidelines on nursing documentation exists (Arko, 2017). Moreover, diverse stakeholders have critiqued nursing documentation in various platforms because of unsatisfactory charting practice. Anecdotal evidence suggests that in some instances, nursing care is not documented or is sub optimal. This surely has serious consequences on assessing care rendered to clients. Also, Asamani et al., (2014) attributed inadequate documentation in a study conducted in Eastern Ghana to inadequate nurse-patient ratio. Another study conducted in Winneba, Ghana, revealed that most nurses did not sign or write their 
initials after documenting care (Okine, 2017). Furthermore, a previous study done in the Tamale Metropolis across five hospitals reported that nursing documentation was very poor (Buunaaisie et al., 2018). Moreover, previous studies on nursing documentation in Ghana including Northern Region are limited in scope as they assessed documentation practices of nurses in both private and public institutions without assessing their knowledge and attitudes towards documentation (Buunaaisie et al., 2018). This current study was conducted to fill the research gap by assessing the knowledge, attitude and practices of documentation among nurses at the Tamale Teaching Hospital (TTH). It also assessed the factors that determine knowledge, attitudes and practices associated with nursing documentation.

\section{Methodology}

\section{Study Design and Setting}

The study employed an analytical cross-sectional design because researchers were able to assess the practice of nursing documentation among nurses and midwives and to relate it to its exposures at the same time. This study was conducted at the TTH because it has numerous speciality areas and nursing documentation was found to be inadequate (Buunaaisie et al., 2018). The hospital is located in Kukuo in the Eastern part of the Tamale Metropolis, Northern Region, Ghana. The hospital has 753 nurses (Adobasom-Anane, 2018). The study was undertaken at the Medical, Paediatric, Surgical and Obstetric Wards of the Hospital.

\section{Study Population}

The target population were qualified registered/professional nurses (i.e., enrolled, diploma or graduate) with at least a year ( $\geq 1$ year) working experience at the selected wards. All participants recruited voluntarily consented to participate in the study. Nurses who were excluded in the study were students and rotational nurses; nurses with less than a year working experience; and nurses on annual or maternity leave. The study selected 288 participants, calculated using the
Yamane Formula(Yamane, 1967) $\quad\left(\mathrm{n}=\frac{N}{1+N(e)^{2}}\right)$, where, $N$ is the population size and $e$, the level of precision. This process was adopted to ensure representativeness of the sample population.

\section{Sampling Procedures}

A 2-stage sampling procedure using first a stratified and then a convenient sampling technique was employed. Stratified sampling ensured equity in the spread of the sample size across purposively selected departments of the Tamale Teaching Hospital which included Medicine, Surgery, Obstetrics and Gynaecology and Special Units. Each department has different wards that formed the sub-clusters. From each department, nurses were conveniently selected from the sub-clusters (wards).

\section{Data Collection Tool and Procedures}

A semi-structured questionnaire was adopted from empirical evidence (Hameed \& Allo, 2014; Asamani et al., 2015; Buunaaisie et al., 2018; Andualem et al., 2019). The instrument comprised five sections; Section A constituted the socio-demographics, Section B was on knowledge and attitudes towards nursing documentation, Section $C$ entailed practices of documentation and Section D constituted factors that influenced nursing documentation. Before actual data collection, pre-testing of the questionnaire was done on 15 nurses at the Tamale Central Hospital but this was not included in the actual data analysis. The questionnaire was later self-administered; hence, respondents were given time to fill the questionnaire and were supervised by data collectors. Respondents were assured of confidentiality and anonymity. This exercise lasted for about 10 minutes and the entire data collection exercise lasted for 2 weeks $\left(17^{\text {th }}-31^{\text {st }}\right.$ August, 2020). Data collectors patiently checked all the completed questionnaire after the data collection exercise to ensure that all fields were completed.

\section{Data Analysis}

In respect of assessing levels of knowledge, attitude and practice of respondents on documentation a number of questions were posed for each variable, and each correct/right choice was scored 1 and 
incorrect/wrong choice scored 0 . The total score for each respondent for each variable was assessed using a composite score by summing all correct answers. The median score for each variable was then computed and used as the cut-off for determining the levels for knowledge (Adequate vs Inadequate), Attitude (Good vs Bad) and Practice (High vs Low). Completed copies of the questionnaire were collected for data inputting and coding. Data was entered and analysed using the Statistical Package for the Social Sciences (SPSS) version 23. Descriptive statistics such as percentages, and frequencies were used to summarize data. A binary logistic regression modelling was done to determine predictors of nursing documentation and the level of significance was set at $\mathrm{p}<0.05$.

\section{Ethical Considerations}

The Research and Ethics Board of the Tamale Teaching Hospital (TTH/R\&D/SR/089) granted ethical approval for the commencement of the study. To ensure ethical standards, informed consent was sought from participants before participation in the study. They were assured of anonymity, privacy and confidentiality.

\section{Results}

Of the 288 copies of the questionnaire distributed, 282 were returned with 279 submitted with valid responses (i.e., more than $90 \%$ of the questions were answered or completed). The response rate was therefore $96.9 \%$. Majority of the participants 157 $(56.3 \%)$ were females and $122(43.7 \%)$ were males. Age characteristics showed that $178(63.8 \%)$ were in the 21-30 years range, $92(33.0 \%)$ in $31-40$ years range and $9(3.2 \%)$ were 41 years and above. Diploma holders were 166 (59.5\%), Degree holders were $75(26.9 \%), 33(11.8 \%)$ had certificates in nursing and $5(1.8 \%)$ had Master's Degree. Regarding nursing category grade, majority of the respondents $173(62.0 \%)$ were Staff Nurses, 72 $(25.8 \%)$ were Nursing Officers and 34 were Enrolled Nurses. With years of experience, majority $200(71.7 \%)$ had worked for 1-5 years, $68(24.4 \%)$ worked for 6-10 years, 9 (3.2\%) worked for 11-15 years and $2(0.7 \%)$ worked for more than 15 years (Table 1).

Table 1: Socio-Demographic Characteristics

\section{$\begin{array}{llll}\text { Variable } & \text { Demographic Characteristics } & \text { Frequency }(n=279) & \text { Percentage }\end{array}$}

\begin{tabular}{|c|c|c|c|}
\hline \multirow[t]{3}{*}{ Sex } & Male & 122 & 43.7 \\
\hline & Female & 157 & 56.3 \\
\hline & Total & 279 & 100.0 \\
\hline \multirow[t]{4}{*}{ Age } & $21-30$ & 178 & 63.8 \\
\hline & $31-40$ & 92 & 33.0 \\
\hline & $>=41$ & 9 & 3.2 \\
\hline & Total & 279 & 100.0 \\
\hline \multirow[t]{3}{*}{ Educational Status } & Certificate & 33 & 11.8 \\
\hline & Diploma & 166 & 59.5 \\
\hline & Degree & 75 & 26.9 \\
\hline
\end{tabular}




\begin{tabular}{|c|c|c|c|}
\hline & Masters & 5 & 1.8 \\
\hline & Total & 279 & 100.0 \\
\hline \multirow[t]{4}{*}{ Grade } & Enrolled Nurse & 33 & 11.8 \\
\hline & Staff Nurse & 174 & 62.4 \\
\hline & Nursing Officer & 72 & 25.8 \\
\hline & Total & 279 & 100.0 \\
\hline \multirow{6}{*}{$\begin{array}{l}\text { Current Work } \\
\text { Setting/Ward }\end{array}$} & Medical & 38 & 13.6 \\
\hline & Surgical & 106 & 38.0 \\
\hline & Paediatric & 30 & 10.8 \\
\hline & Obstetric & 89 & 31.9 \\
\hline & Special Units & 16 & 5.7 \\
\hline & Total & 279 & 100.0 \\
\hline \multirow[t]{5}{*}{ Years of experience } & $1-5$ years & 200 & 71.7 \\
\hline & $6-10$ years & 68 & 24.4 \\
\hline & $11-15$ years & 9 & 3.2 \\
\hline & $>15$ years & 2 & 0.7 \\
\hline & Total & 279 & 100.0 \\
\hline
\end{tabular}

Source: Field survey, 2020

Level of knowledge regarding nursing care documentation

In this study, adequate knowledge was adjudged at a score of 7 and above on the knowledge component of the questionnaire. Inadequate knowledge consequently represents any score less than 7 . The analyses revealed that $236(84.6 \%)$ had adequate knowledge on nursing documentation whilst 43 (15.4\%) had inadequate knowledge. Respondents were then quizzed on their source of knowledge on nursing documentation. Majority of the respondents 188 (67.4\%) had their knowledge from Nursing School, 52 (18.6\%) from Nurses on the ward, 13 (4.7\%) from Hospital Management and 26 (9.3\%) from other nurses' notes (Table 2). 
Table 2: Level of Knowledge on Nursing Documentation

$\begin{array}{lll}\text { Variables } & \text { Frequency } & \text { Percentage }\end{array}$

\begin{tabular}{|c|c|c|c|}
\hline \multirow[t]{3}{*}{ Knowledge } & Adequate knowledge & 236 & 84.6 \\
\hline & Inadequate knowledge & 43 & 15.4 \\
\hline & Total & 279 & 100.0 \\
\hline \multirow[t]{5}{*}{ Sources of knowledge } & Nursing School & 188 & 67.4 \\
\hline & Nurses on the ward & 52 & 18.6 \\
\hline & Hospital Management & 13 & 4.7 \\
\hline & From other Nurses Notes & 26 & 9.3 \\
\hline & Total & 279 & 100.0 \\
\hline \multirow[t]{6}{*}{$\begin{array}{l}\text { Knowledge on } \\
\text { definition }\end{array}$} & $\begin{array}{l}\text { Recording data including patient care } \\
\text { management }\end{array}$ & 7 & 3.2 \\
\hline & Recording nursing care & 10 & 4.6 \\
\hline & Stating what is done on the patient in the folder & 6 & 2.7 \\
\hline & $\begin{array}{l}\text { Writing procedures done on the patient in the } \\
\text { folder }\end{array}$ & 188 & 85.8 \\
\hline & $\begin{array}{l}\text { Written document that signifies what was care } \\
\text { given to a patient }\end{array}$ & 8 & 3.7 \\
\hline & Total & 219 & 100.0 \\
\hline \multirow{6}{*}{$\begin{array}{l}\text { Knowledge on } \\
\text { potential } \\
\text { consequences of } \\
\text { inadequate } \\
\text { documentation }\end{array}$} & Legal implications & 79 & 43.6 \\
\hline & Medication/Treatment errors & 18 & 9.9 \\
\hline & No continuity of care & 35 & 19.3 \\
\hline & Poor patient outcomes & 44 & 24.3 \\
\hline & Miscommunication among the health team & 5.0 & 2.8 \\
\hline & Total & 181 & 100.0 \\
\hline \multicolumn{2}{|c|}{ Documentation is an important function of professional nursing practice } & 276 & 98.9 \\
\hline \multicolumn{2}{|c|}{ Documentation must be done according to set guidelines } & 237 & 84.9 \\
\hline \multicolumn{2}{|c|}{ Documentation must necessarily be factual, accurate, legible and organized } & 276 & 98.9 \\
\hline \multicolumn{2}{|c|}{ Documentation entails what I have observed and done } & 261 & 93.5 \\
\hline
\end{tabular}




\begin{tabular}{lcc}
\hline Documentation includes what I think & 137 & 49.1 \\
\hline I know that documentation has legal implications & 262 & 93.9 \\
\hline I know that documentation ensure continuity of care & 262 & 93.9 \\
\hline
\end{tabular}

Source: Field survey, 2020

The definition of nursing documentation was inquired of the participants. Majority of those that responded; 200 $(69.9 \%)$ had their answers centred around "Nursing documentation involves writing procedures done on the patient in the folder". Almost all of the respondents 276 (98.9\%) were of the impression that documentation is an important function of professional nursing practice. Similar percentage of respondents knew documentation must necessarily be factual, accurate, legible and organised. Majority of participants 237 (84.9\%) knew documentation must be done according to set guidelines; but only a few; 40 (14.3\%) had no idea. Again, 261 (93.5\%) of respondents knew documentation entails what is observed and done while $16(5.7 \%)$ did not know this. As high as 274 (98.2\%) knew documentation has legal implications and $3(1.1 \%)$ did not know. Also, 262 (93.9\%) of the participants knew documentation ensured continuity of care while $15(5.4 \%)$ were oblivious. With the variable "Documentation includes what I think", opinion was split almost equally with 137 (49.1\%) affirming to the statement and 140 (50.2\%) denying knowledge of the statement. Regarding respondents' knowledge on the potential consequences of inadequate documentation, 98 (35.1\%) left the question unanswered and 181 (64.9\%) answered the question correctly. Responses mainly centred on legal implications, poor patient outcomes, no continuity of care, medication/treatment errors and miscommunication among the health team with 79 (43.6\%), 44 (24.3\%), 35 (19.3\%), 18 (9.9\%) and 5 (2.8\%) responses respectively (Table 2).

Inferential analysis done revealed that respondents between 21-30 years were less likely to have adequate knowledge on nursing documentation (AOR 0.12, 95\% C1: 0.029-1.507; $\mathrm{p}=0.004$ ) compared to their younger counterparts (Table 3). Also, respondents who had between 1 to 5 years of working experience were more likely to have adequate knowledge on nursing documentation (AOR 15.29, 95\% C1: 3.083-75.872, $\mathrm{p}=0.001$ ) as compared to their counterparts with longer working experience.

Table 3: Association between socio-demographic characteristics and level of knowledge

Variable

SocioLevel of knowledge

Total AOR (CI95\%)

p-value demographic characteristics Adequate Inadequate Knowledgeable Knowledgeable

\begin{tabular}{|c|c|c|c|c|c|c|}
\hline \multirow[t]{3}{*}{ Age } & $21-30$ & $142(79.8 \%)$ & $36(20.2 \%)$ & 178 & $0.12(0.029-1.507)$ & $0.004 *$ \\
\hline & $31-40$ & $85(92.4 \%)$ & $7(7.6 \%)$ & 92 & - & 1.00 \\
\hline & $40+$ & $9(100.0 \%)$ & $0(0.0 \%)$ & 9 & 1.00 & \\
\hline \multirow[t]{3}{*}{ Gender } & Male & $112(91.8 \%)$ & $10(8.2 \%)$ & 122 & $1.17(0.438-3.121)$ & 0.756 \\
\hline & Female & $124(79.0 \%)$ & $33(21.0 \%)$ & 157 & 1.00 & \\
\hline & Certificate & $30(90.9 \%)$ & $3(9.1 \%)$ & 33 & 1.00 & 1.00 \\
\hline Seidu, et a & \multicolumn{5}{|c|}{ DOI: https://doi.org.10.47740/567.UDSIJD6i } & 546 \\
\hline
\end{tabular}




\begin{tabular}{|c|c|c|c|c|c|c|}
\hline \multirow{3}{*}{$\begin{array}{l}\text { Educational } \\
\text { status }\end{array}$} & Diploma & $130(78.3 \%)$ & $36(21.7 \%)$ & 166 & $0.34(0.044-2.65)$ & 0.304 \\
\hline & Degree & $71(94.7 \%)$ & $4(5.3 \%)$ & 75 & 1.87 & 1.00 \\
\hline & Masters & $5(100.0 \%)$ & $0(0.0 \%)$ & 5 & 1.00 & \\
\hline \multirow[t]{3}{*}{ Grade } & Enrolled Nurse & $30(90.9 \%)$ & $3(9.1 \%)$ & 33 & 1.00 & 1.00 \\
\hline & Staff Nurse & $138(79.3 \%)$ & $36(20.7 \%)$ & 174 & 1.00 & - \\
\hline & $\begin{array}{l}\text { Nursing } \\
\text { Officer }\end{array}$ & $68(94.4 \%)$ & $4(5.6 \%)$ & 72 & 1.00 & \\
\hline \multirow{5}{*}{$\begin{array}{l}\text { Current work } \\
\text { setting/ward }\end{array}$} & Medical & $36(94.7 \%)$ & $2(5.3 \%)$ & 38 & $0.90(0.172-4.738)$ & 0.903 \\
\hline & Surgical & $98(92.5 \%)$ & $8(7.5 \%)$ & 106 & $5.32(0.844-33.593)$ & 0.075 \\
\hline & Paediatric & $25(83.3 \%)$ & $5(16.7 \%)$ & 30 & $4.05(0.793-20.668)$ & 0.093 \\
\hline & Obstetric & $63(70.8 \%)$ & $26(29.2 \%)$ & 89 & $1.81(0.215-15.188)$ & 0.586 \\
\hline & Special Units & $14(87.5 \%)$ & $2(12.5 \%)$ & 16 & 1.00 & \\
\hline \multirow{4}{*}{$\begin{array}{l}\text { Years of } \\
\text { experience }\end{array}$} & $1-5$ years & $170(85.0 \%)$ & $30(15.0 \%)$ & 200 & $15.29(3.083-75.872$ & 0.001* \\
\hline & $6-10$ years & $55(80.9 \%)$ & $13(19.1 \%)$ & 68 & - & 0.999 \\
\hline & $11-15$ years & $9(100.0 \%)$ & $0(0.0 \%)$ & 9 & - & 1.00 \\
\hline & $>15$ years & $2(100.0 \%)$ & $0(0.0 \%)$ & 2 & - & - \\
\hline
\end{tabular}

Source: Field survey, 2020

Attitudes Regarding Nursing Documentation

Positive attitude was adjudged at a score of 4 and above on the attitude component of the questionnaire. Negative attitude consequently was represented by any score less than 4 . Majority of the respondents $(84.2 \%)$ had a positive attitude towards nursing documentation (Table 4). In particular, majority of the respondents agreed that nursing documentation is as important as any other patient related documentation, nursing notes are meaningful and they like documenting activities in the ward, nurses possess sufficient knowledge on nursing documentation and they always make their handwriting legible. Also, cumulatively, 187 $(67.0 \%)$ agreed that their documentation depends on how familiar they are with documentation policies and $92(33.0 \%)$ disagreed. Regarding the variable "I document for handing-over purposes only", 66 (23.7\%) strongly disagreed, $126(45.2 \%)$ disagreed, $32(11.5 \%)$ agreed and $55(19.7 \%)$ strongly agreed. Based on the variable "I like documenting my activities in the ward", $263(94.3 \%)$ of the respondents were considered to have good attitudes towards nursing documentation (Table 5). Males were more likely to have a positive attitude towards nursing documentation than females (AOR, $2.81 \mathrm{CI}$, 1.434-5.501, $\mathrm{p}=0.003$ ). Respondents in the medical, surgical and paediatric wards had positive attitudes towards nursing documentation as compared to their counterparts in the other wards (Table 6). 
Table 4: Attitude score on Nursing Documentation
Attitudes
Frequency $(\mathbf{n}=279)$
Percentage

\begin{tabular}{lcc}
\hline Positive attitudes & 235 & 84.2 \\
\hline Negative attitudes & 44 & 15.8 \\
\hline Total & 279 & 100.0 \\
\hline
\end{tabular}

Source: Field survey, 2020

Table 5: Respondents' attitudes towards Nursing Documentation

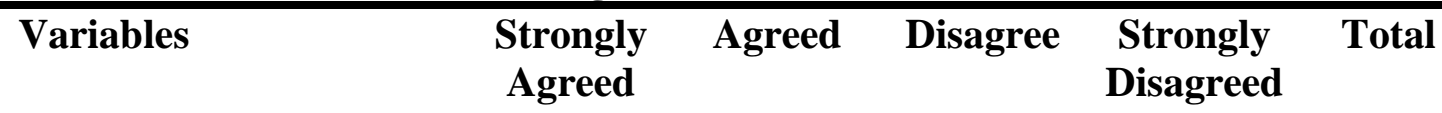

\begin{tabular}{|c|c|c|c|c|c|}
\hline $\begin{array}{l}\text { Nursing documentation is as important } \\
\text { as any other patient related } \\
\text { documentation }\end{array}$ & $\begin{array}{c}214 \\
(76.7 \%)\end{array}$ & $\begin{array}{c}64 \\
(22.9 \%)\end{array}$ & $\begin{array}{c}1 \\
(0.4 \%)\end{array}$ & $\begin{array}{c}0 \\
(0.0 \%)\end{array}$ & $\begin{array}{r}279 \\
(100)\end{array}$ \\
\hline $\begin{array}{l}\text { Nursing notes are meaningful and a } \\
\text { nursing priority }\end{array}$ & $\begin{array}{c}165 \\
(59.1 \%)\end{array}$ & $\begin{array}{c}110 \\
(39.4 \%)\end{array}$ & $\begin{array}{c}4 \\
(1.4 \%)\end{array}$ & $\begin{array}{c}0 \\
(0.0 \%)\end{array}$ & $\begin{array}{r}279 \\
(100)\end{array}$ \\
\hline $\begin{array}{l}\text { My documentation depends on how } \\
\text { familiar I am to documentation policies }\end{array}$ & $\begin{array}{c}72 \\
(25.8 \%)\end{array}$ & $\begin{array}{c}115 \\
(41.2 \%)\end{array}$ & $\begin{array}{c}82 \\
(29.4 \%)\end{array}$ & $10(3.6 \%)$ & $\begin{array}{r}279 \\
(100)\end{array}$ \\
\hline $\begin{array}{l}\text { I like documenting my activities in the } \\
\text { ward }\end{array}$ & $\begin{array}{c}130 \\
(46.6 \%)\end{array}$ & $\begin{array}{c}133 \\
(47.7 \%)\end{array}$ & $\begin{array}{c}16 \\
(5.7 \%)\end{array}$ & $\begin{array}{c}0 \\
(0.0 \%)\end{array}$ & $\begin{array}{r}279 \\
(\mathbf{1 0 0 )}\end{array}$ \\
\hline $\begin{array}{l}\text { I document for handing-over purposes } \\
\text { only }\end{array}$ & $\begin{array}{c}55 \\
(19.7 \%)\end{array}$ & $\begin{array}{c}32 \\
(11.5 \%)\end{array}$ & $\begin{array}{c}126 \\
(45.2 \%)\end{array}$ & $\begin{array}{c}66 \\
(23.7 \%)\end{array}$ & $\begin{array}{l}279 \\
(100)\end{array}$ \\
\hline $\begin{array}{l}\text { Nurses possess sufficient knowledge on } \\
\text { documentation }\end{array}$ & $\begin{array}{c}89 \\
(31.9 \%)\end{array}$ & $\begin{array}{c}161 \\
(57.7 \%)\end{array}$ & $\begin{array}{c}29 \\
(10.4 \%)\end{array}$ & $\begin{array}{c}0 \\
(0.0 \%)\end{array}$ & $\begin{array}{r}279 \\
(100)\end{array}$ \\
\hline I make my handwriting legible & $\begin{array}{c}117 \\
(41.9 \%)\end{array}$ & $\begin{array}{c}153 \\
(54.8 \%)\end{array}$ & $\begin{array}{c}7 \\
(2.5 \%)\end{array}$ & $\begin{array}{c}2 \\
(0.7 \%)\end{array}$ & $\begin{array}{r}279 \\
(100)\end{array}$ \\
\hline
\end{tabular}

Source: Field survey, 2020 
Table 6: Association between socio-demographic characteristics and attitudes towards nursing documentation

\begin{tabular}{|c|c|c|c|c|c|c|}
\hline \multirow[t]{2}{*}{ Variable } & \multirow{2}{*}{$\begin{array}{c}\text { Socio- } \\
\text { demographic } \\
\text { characteristics }\end{array}$} & \multicolumn{2}{|c|}{ Attitude } & \multirow[t]{2}{*}{ Total } & \multirow[t]{2}{*}{ AOR (CI95\%) } & \multirow[t]{2}{*}{ p-value } \\
\hline & & $\begin{array}{l}\text { Positive } \\
\text { attitude }\end{array}$ & $\begin{array}{l}\text { Negative } \\
\text { attitude }\end{array}$ & & & \\
\hline \multirow[t]{3}{*}{ Age } & $21-30$ & $55(30.9 \%)$ & $123(69.1 \%)$ & 178 & $1.91(0.947-3.861)$ & 0.070 \\
\hline & $31-40$ & $43(46.7 \%)$ & $49(53.3 \%)$ & 92 & 1.00 & \\
\hline & $40+$ & $2(22.2 \%)$ & $7(77.8 \%)$ & 9 & 1.00 & \\
\hline \multirow[t]{2}{*}{ Sex } & Male & $35(28.7 \%)$ & $87(71.3 \%)$ & 122 & $2.81(1.434-5.501)$ & 0.003* \\
\hline & Female & $65(41.4 \%)$ & $92(58.6 \%)$ & 157 & 1.00 & \\
\hline \multirow{4}{*}{$\begin{array}{l}\text { Educational } \\
\text { status }\end{array}$} & Certificate & $12(36.4 \%)$ & $21(63.6 \%)$ & 33 & 1.00 & \\
\hline & Diploma & $54(32.5 \%)$ & $112(67.5 \%)$ & 166 & $0.91(0.276-3.002)$ & 0.878 \\
\hline & Degree & $32(42.7 \%)$ & $43(57.3 \%)$ & 75 & 1.00 & \\
\hline & Masters & $2(40.0 \%)$ & $3(60.0 \%)$ & 5 & 1.00 & \\
\hline \multirow[t]{3}{*}{ Grade } & Enrolled Nurse & $12(36.4 \%)$ & $21(63.6 \%)$ & 33 & 1.00 & \\
\hline & Staff Nurse & $54(31.0 \%)$ & $120(69.0 \%)$ & 174 & 1.00 & \\
\hline & Nursing Officer & $34(47.2 \%)$ & $38(52.8 \%)$ & 72 & 1.00 & \\
\hline \multirow{5}{*}{$\begin{array}{l}\text { Current } \\
\text { work } \\
\text { setting/ward }\end{array}$} & Medical & $22(57.9 \%)$ & $16(42.1)$ & 38 & $0.29(0.121-0.694)$ & 0.005* \\
\hline & Surgical & $34(32.1 \%)$ & $72(67.9 \%)$ & 106 & $0.17(0.057-0.534)$ & $0.002 *$ \\
\hline & Paediatric & $9(30.0 \%)$ & $21(70.0 \%)$ & 30 & $0.19(0.072-0.513)$ & $0.001 *$ \\
\hline & Obstetric & $30(33.7 \%)$ & $59(66.3 \%)$ & 89 & $0.26(0.068-1.015)$ & 0.053 \\
\hline & Special Units & $5(31.3 \%)$ & $11(68.8 \%)$ & 16 & 1.00 & \\
\hline \multirow{4}{*}{$\begin{array}{l}\text { Years of } \\
\text { experience }\end{array}$} & $1-5$ years & $64(32.0 \%)$ & $136(68.0 \%)$ & 200 & $1.81(0.744-4.376)$ & 0.191 \\
\hline & 6-10 years & $34(5.0 \%)$ & $34(50.0 \%)$ & 68 & 1.00 & \\
\hline & $11-15$ years & $2(22.0 \%)$ & $7(77.8 \%)$ & 9 & 1.00 & \\
\hline & Greater than 15 & $0(0.0 \%)$ & $2(100.0 \%)$ & 2 & 1.00 & \\
\hline
\end{tabular}

Source: Field survey, 2020 
Documentation Practice by nurses

A large number of the participants 215 (77.1\%) asserted that they documented always, $62(22.2 \%)$ documented sometimes and $2(0.7 \%)$ rarely documented. Of the respondents who "always" documented care on patients, $74 \%$ were deemed to have adequate practice, whilst the rest $(24.0 \%)$ had inadequate practice. On documentation of education and advice given to patients, less than half 131 (47.0\%) did it always and 107 (38.4\%) documented sometimes. Procedures that do not require charting were also documented always by 137 (49.1\%) of the respondents whilst 103 (36.9\%) documented sometimes (Table 7).

Also, majority $229 \quad(82.1 \%)$ documented immediately after the care is rendered, $43(15.4 \%)$ documented anytime when convenient and 7 (2.5\%) documented at the end of shift hours. Regarding correction of documentation errors, majority of the participants $224(82.4 \%)$ strike through, signed and moved to a new line, 46 (16.9\%) used correction fluid and $2(0.7 \%)$ just ignored the error (Table 7$)$.

On some of the intricacies of documentation, all of the respondents $(100.0 \%)$ affirmed they always write time and date during documentation. Majority of respondents $182(65.2 \%)$ report medical error in documentation voluntarily and 97 (34.8\%) do not. In the case of documenting patients' response to care, $235(84.2 \%)$ documented while $44(15.8 \%)$ do not. Also, 247 (88.5\%) sign nurses notes with names or initials whilst $32(11.5 \%)$ do not. Furthermore, majority of the participants $241(86.4 \%)$ do not use abbreviations and shorthand during documentation (Table 7). In addition, majority of the respondents, $273(97.8 \%)$ read their colleagues notes whilst 6 (2.2\%) do not. Also, 208 (74.6\%) of the participants asserted that their colleague nurses' notes fulfilled standards of nursing whilst 71 (25.4\%) denied it. With regard to electronic documentation, majority 179 (64.2\%) have not used a computerised documentation system whilst 100 (35.8\%) have used computers for nursing documentation (Table 7). Respondents aged 21-30 years were shown to have adequate level of practice of nursing documentation (AOR, 5.85 (2.64-12.97), $\mathrm{p}<0.001)$. Certificate nurses significantly had adequate practice of nursing documentation (23.79 (2.23-253.25), $\mathrm{p}=0.009)$ (Table 8). Nurses in the surgical wards, paediatric and obstetric wards had adequate practice of documentation. Respondents who had adequate knowledge were also significantly more likely to have adequate practice of nursing documentation (Table 9).

Table 7: Practice of Nursing Documentation

\begin{tabular}{llccc}
\hline & \multicolumn{1}{c}{ Variables } & Frequency & Percentage \\
\hline Practice & Adequate practice & 159 & 74.1 \\
& Inadequate practice & 56 & 22.9 \\
\cline { 2 - 5 } & & $\mathbf{2 1 5}$ & $\mathbf{1 0 0 . 0}$ \\
\hline $\begin{array}{l}\text { Nursing } \\
\text { documentation for } \\
\text { every patient }\end{array}$ & Always & Total & 215 & 77.1 \\
& Sometimes & 62 & 22.2 \\
& Rarely & 2 & 0.7 \\
& Never & & 0 & 0.0 \\
\cline { 2 - 5 } & & Total & $\mathbf{2 7 9}$ & $\mathbf{1 0 0 . 0}$ \\
\cline { 2 - 4 }
\end{tabular}




\begin{tabular}{|c|c|c|c|}
\hline \multirow{5}{*}{$\begin{array}{l}\text { Documents education } \\
\text { or advice given }\end{array}$} & Always & 131 & 47.0 \\
\hline & Sometimes & 107 & 37 \\
\hline & Rarely & 37 & 13.3 \\
\hline & Never & 4 & 1.4 \\
\hline & Total & 279 & 100.0 \\
\hline \multirow{5}{*}{$\begin{array}{l}\text { Documents other } \\
\text { procedures that don't } \\
\text { require charting (Eg. } \\
\text { Wound dressing) }\end{array}$} & Always & 137 & 49.1 \\
\hline & Sometimes & 103 & 36.9 \\
\hline & Rarely & 32 & 11.5 \\
\hline & Never & 7 & 2.5 \\
\hline & Total & 279 & 100.0 \\
\hline \multirow{4}{*}{$\begin{array}{l}\text { Time preference to } \\
\text { document }\end{array}$} & Anytime when convenient & 43 & 15 \\
\hline & Immediately after care is rendered & 229 & 82.1 \\
\hline & At the end of shift hours & 7 & 2.5 \\
\hline & Total & 279 & 100.0 \\
\hline \multirow{4}{*}{$\begin{array}{l}\text { Correction of } \\
\text { documentation errors }\end{array}$} & Strikethrough and signed & 224 & 82.4 \\
\hline & Used correction fluid & 46 & 16.9 \\
\hline & Ignored error & 2 & 0.7 \\
\hline & Total & 272 & 100.0 \\
\hline \multicolumn{2}{|c|}{ I always write time and date during documentation } & 279 & 100.0 \\
\hline \multicolumn{2}{|l|}{ Read colleagues notes } & 273 & 97.8 \\
\hline \multicolumn{2}{|c|}{ Colleagues note fulfil standards } & 208 & 74.6 \\
\hline \multicolumn{2}{|c|}{ Uses computerized documentation system } & 100 & 35.8 \\
\hline \multicolumn{2}{|c|}{ Reports medical error in documentation voluntarily } & 182 & 65.2 \\
\hline \multicolumn{2}{|c|}{ Document patient's response to care } & 235 & 84.2 \\
\hline \multicolumn{2}{|c|}{ Sign notes with names or initials } & 247 & 88.5 \\
\hline \multicolumn{2}{|c|}{ I typically use abbreviations and short hands } & 38 & 13.6 \\
\hline
\end{tabular}

Source: Field survey, 2020

Seidu, et al., 2021: UDSIJD Vol 8(1) 
Table 8: Relationship between socio-demographic characteristics and level of practice of nursing documentation.

\begin{tabular}{|c|c|c|c|c|c|c|}
\hline \multirow[t]{2}{*}{ Variable } & \multirow{2}{*}{$\begin{array}{c}\text { Socio- } \\
\text { demographic } \\
\text { characteristics }\end{array}$} & \multicolumn{2}{|c|}{ Level of practice } & \multirow[t]{2}{*}{ Total } & \multirow[t]{2}{*}{ AOR (CI95\%) } & \multirow[t]{2}{*}{ p-value } \\
\hline & & $\begin{array}{c}\text { Adequate } \\
\text { practice }\end{array}$ & $\begin{array}{c}\text { Inadequate } \\
\text { practice }\end{array}$ & & & \\
\hline \multirow[t]{3}{*}{ Age } & $21-30$ & $154(86.5 \%)$ & $24(13.5 \%)$ & 178 & $5.85(2.637-12.974$ & $<0.001^{*}$ \\
\hline & $31-40$ & $54(58.7 \%)$ & $38(41.3 \%)$ & 92 & 1.00 & \\
\hline & $40+$ & $7(77.8 \%)$ & $2(22.2 \%)$ & 9 & 1.00 & \\
\hline \multirow[t]{2}{*}{ Sex } & Male & $90(73.8 \%)$ & $32(26.2 \%)$ & 122 & $1.46(0.75-3.150)$ & 0.337 \\
\hline & Female & $125(79.6 \%)$ & $32(20.4 \%)$ & 157 & 1.00 & \\
\hline \multirow{4}{*}{$\begin{array}{l}\text { Educational } \\
\text { status }\end{array}$} & Certificate & $26(78.8 \%)$ & $7(21.2 \%)$ & 33 & $0.30(0.002-0.408)$ & $0.008^{*}$ \\
\hline & Diploma & $134(80.7 \%)$ & $32(19.3 \%)$ & 166 & $0.49(0.122-1.956)$ & 0.311 \\
\hline & Degree & $52(69.3 \%)$ & $23(30.7 \%)$ & 75 & 1.00 & \\
\hline & Masters & $3(60.0 \%)$ & $2(40.0 \%)$ & 5 & 1.00 & \\
\hline \multirow[t]{3}{*}{ Grade } & Enrolled Nurse & $26(78.8 \%)$ & $7(21.2 \%)$ & 33 & $23.79(2.234-253.249)$ & 0.009* \\
\hline & Staff Nurse & $135(77.6 \%)$ & $39(22.4 \%)$ & 174 & 1.00 & \\
\hline & Nursing Officer & $54(75.0 \%)$ & $18(25.0 \%)$ & 72 & 1.00 & \\
\hline \multirow{5}{*}{$\begin{array}{l}\text { Current work } \\
\text { setting/ward }\end{array}$} & Medical & $22(57.9 \%)$ & $16(42.1 \%)$ & 38 & $.410(0.165-1.019)$ & 0.055 \\
\hline & Surgical & $80(75.5 \%)$ & $26(24.5 \%)$ & 106 & $0.25(0.071-0.872)$ & $0.030 *$ \\
\hline & Paediatric & $25(83.3 \%)$ & $5(16.7 \%)$ & 30 & $0.27(0.095-0.791)$ & $0.017 *$ \\
\hline & Obstetric & $73(82.0 \%)$ & $16(18.0 \%)$ & 89 & $0.11(0.012-0.995)$ & $0.050 *$ \\
\hline & Special Units & $15(93.8 \%)$ & $1(6.3 \%)$ & 16 & 1.00 & \\
\hline \multirow{4}{*}{$\begin{array}{l}\text { Years of } \\
\text { experience }\end{array}$} & $1-5$ years & $161(80.5 \%)$ & $39(19.5 \%)$ & 200 & $0.866(0.316-2.374)$ & 0.780 \\
\hline & $6-10$ years & $45(66.2 \%)$ & $23(33.8 \%)$ & 68 & 1.00 & \\
\hline & $11-15$ years & $9(100.0 \%)$ & $0(0.0 \%)$ & 9 & 1.00 & \\
\hline & Greater than 15 & $0(0.0 \%)$ & $2(100.0 \%)$ & 2 & 1.00 & \\
\hline
\end{tabular}

Source: Field survey, 2020 
Table 9: Association between level of knowledge and practice of nursing documentation

\begin{tabular}{|c|c|c|c|c|c|}
\hline \multirow[t]{2}{*}{ Level of practice } & \multicolumn{2}{|c|}{ Level of knowledge } & \multirow[t]{2}{*}{ Total } & \multirow[t]{2}{*}{ COR (CI 95\%) } & \multirow[t]{2}{*}{ P-value } \\
\hline & $\begin{array}{c}\text { Adequate } \\
\text { knowledge }\end{array}$ & $\begin{array}{l}\text { Inadequate } \\
\text { knowledge }\end{array}$ & & & \\
\hline Adequate practice & $174(80.9 \%)$ & $41(19.1 \%)$ & 215 & $0.137(0.032-0.583)$ & $0.007 *$ \\
\hline Inadequate practice & $62(96.9 \%)$ & $2(3.1 \%)$ & 64 & 1.00 & \\
\hline
\end{tabular}

Source: Field survey, 2020

Organizational Factors that influenced Nursing Documentation

When the respondents were presented with the task of specifying the organizational factors that could influence or impact on documentation practice, a large number, $198(71.0 \%)$ mentioned 'availability of operational standards for nursing care documentation' as one of the factors. More than half, 167 (59.9\%) affirmed 'in-service training given within the facility' on documentation. In addition, majority, $214(76.7 \%)$ confirmed 'lack of adequate staff' in the facility as a factor that influenced documentation. Majority, 180 (64.5\%) also affirmed 'no motivation from supervisors' as a factor that influenced the practice of nursing documentation. Regarding the 'availability of documentation sheets', 150 (53.8\%) affirmed the existence of documenting sheets in the facility influenced documentation. Furthermore, 150 (53.8\%) stated the 'lack of nursing care plan sheets', as well as 'Nurses' apathy towards documentation' $(175 ; 62.7 \%)$ were influencing factors to documentation practice (Table $10)$.

Table 10: Organizational Factors that influenced Nursing Documentation

$\begin{array}{llll}\text { Organizational Factors } & \text { Yes } & \text { No } & \text { Total }\end{array}$

\begin{tabular}{|c|c|c|c|}
\hline $\begin{array}{l}\text { Availability of operational standard for nursing } \\
\text { care documentation }\end{array}$ & $198(71.0 \%)$ & $81(29.0 \%)$ & $279(100.0)$ \\
\hline In-service training on documentation & $167(59.9 \%)$ & $112(40.1 \%)$ & $279(100.0)$ \\
\hline $\begin{array}{l}\text { No adequate staff (Higher nurse-patient ratio)/ } \\
\text { Increased work-load }\end{array}$ & $214(76.7 \%)$ & $65(23.3 \%)$ & $279(100.0)$ \\
\hline Availability of nursing care plan sheet & $114(40.9 \%)$ & $165(59.1 \%)$ & $279(100.0)$ \\
\hline Inadequate documenting sheets & $129(46.2 \%)$ & $150(53.8 \%)$ & $279(100.0)$ \\
\hline $\begin{array}{l}\text { Unfamiliarity with standard of nursing } \\
\text { documentation }\end{array}$ & $139(49.8 \%)$ & $140(50.2 \%)$ & $279(100.0)$ \\
\hline No obligation from the hospital & $155(55.6 \%)$ & $124(44.4 \%)$ & $279(100.0)$ \\
\hline No motivation from supervisors & $180(64.5 \%)$ & $99(35.5 \%)$ & $279(100.0)$ \\
\hline Unsatisfied monthly salary & $128(45.9 \%)$ & $151(54.1 \%)$ & $279(100.0)$ \\
\hline
\end{tabular}

Source: Field survey, 2020 


\section{Discussion}

The study revealed that majority of respondents $(84.6 \%)$ had adequate knowledge on nursing documentation. The high level of knowledge in this study could be attributed to the fact that majority of participants had less than 5 years of experience and it could be inferred that nursing documentation knowledge acquired in nursing school could still be fresh in their minds. With high knowledge, nurses will be motivated to document care rendered to clients (Karkkainen et al., 2005; Johnson,2011). Previous studies in five facilities in the Tamale metropolis contradicted this finding with a revelation that nurses' level of knowledge on documentation was poor (Buunaaisie et al., 2018). In this current study, knowledge assessment was on self-report basis unlike that study of Buunaaisie et al., (2018) which was retrospective in nature. This could account for the disparity in knowledge results. In contrast with other studies elsewhere in Africa, Tasew et al., (2019) reported poor knowledge for majority of respondents in Ethiopia and Hassan et al., (2018) also reported inadequate knowledge about documentation in Egypt. The main source of knowledge of the participants was nursing school. This finding was consistent with studies conducted among nurses in Nigeria (Taiye, 2015) and Ethiopia (Andualem et al., 2019) where the major source of information about documentation was nursing school. Almost all of the respondents were of the impression that documentation is an important function of professional nursing practice that promotes continuity of care, must necessarily be factual, accurate, legible and organised and must be done according to set guidelines. Andualem et al. (2019) reported similar findings in a cross-sectional study conducted among nurses in Ethiopia. Participants (35.1\%) in this current study also showed sufficient knowledge on the potential consequences of inadequate documentation that include legal implications, poor patient outcomes, no continuity of care and medication/treatment errors. Andualem et al., (2019) noted that among all study participants, just $8.3 \%$ of them did not know about the potential consequences of inadequate documentation. This wide difference could be accounted for by the difference in sample size of both studies. Inferential analysis done revealed that respondents between 21-30 years were more knowledgeable on nursing documentation. This could be due to the fact that nurses of younger age are fresher from nursing training schools and may possess more knowledge. Also, respondents who had between 1 to 5 years of working experience had adequate knowledge on nursing documentation. This follows those nurses with 1 to 5 years in nursing practice are typically younger and completed nursing school few years ago. This may have accounted for younger nurses and those with less than 5 years of experience having adequate knowledge.

Several authors have suggested that competence and attitudes of nurses relate to their intention to document and sometimes act as a setback to quality documentation (Allen, 1998; Karkkainen et al., 2005; Johnson, 2011). In the daily practice of nursing documentation, attitudes of nurses is of immense significance (Bjorvell, 2002). In this study, majority of the study participants; above $80 \%$ were more positive in their attitudes towards documentation and over $90 \%$ agreed that nursing documentation is as important as any other patient related documentation, and nursing notes are meaningful and a nursing priority. The participants also disclosed that they liked documenting activities in the ward, and believed that nurses possess sufficient knowledge on nursing documentation and they always make their handwriting legible. These findings were congruent with a study which also found that nurses strongly agreed that nursing notes were meaningful and necessary for legal protection, as well as a nursing priority (Nakate et al., 2015). Andualem et al., (2019) also found that majority of nurses in Ethiopia strongly agreed on equal importance of nursing care documentation as any other patient documentation and the importance of nursing care documentation to other health care providers. Similarly, findings from Taiye (2015) has 
confirmed that their respondents liked documenting their activities (Taiye, 2015) Males had positive attitudes towards nursing documentation than females. Male nurses usually tend to stay at work throughout the shift and are liable to scrutinize documentation at the end of the shift. Some female nurses on the other hand may be breastfeeding mothers or pregnant; and these tend to close work earlier and won't answer for their documentation errors at the end of their shift. This may account for males' better attitudes towards documentation. Respondents in the medical, surgical and paediatric wards had positive attitudes towards nursing documentation as compared to their counterparts in the other wards. In the medical, surgical and paediatric wards, there are many nursing procedures that demand documentation as opposed to wards such as speciality clinics where nursing procedures may be limited.

The practice of documentation has been discussed as important to the delivery of health services and improvements in treatment outcomes (Mathioudakis et al., 2016). In this study, majority of the participants $(77.1 \%)$ asserted that they documented always. This assessment was based on frequency of documentation but not its quality. This finding differed markedly from an earlier study conducted in several hospitals in Tamale including the TTH where the practice of documentation of nurses in the hospitals selected was very poor (Buunaaisie et al., 2018). The difference stemmed from the difference in documentation practice assessment approach; the latter used a retrospective study of patient folders while the former used self-reported questionnaire. In other studies, (Tasew et al., 2019) reported inadequate practice of nursing care documentation in Ethiopia and Andualem et al. (2019) also reported that majority of participants in the study had poor nursing care documentation practice. This finding is consistent with that of a previous study conducted in the Tamale metropolis which revealed that specific procedures such as wound dressing were hardly documented (Buunaaisie et al., 2018).
Concerning time preference when documenting, majority preferred to document immediately after the care is rendered. This finding is consistent with a cross-sectional study conducted among nurses in Ethiopia, where majority of the respondents mostly documented immediately after the care was rendered (Andualem et al., 2019). On the correction of documentation errors, majority of the participants $(80.3 \%)$ strikethrough, signed and moved to a new line. This finding differed from a study conducted in Winneba, Ghana where majority of nurses were found not to sign or write their initials after documenting care (Okine, 2017). The disparity stemmed from the fact that Okine, (2017) studied patient folders retrospectively unlike the self-report approach of this current study. Results showed that few of the nurses do use computerised documentation system. Similarly, a study in Ethiopia among nurses showed few nurses reported that they used computerized nursing care documentation system (Andualem et al., 2019). Studies suggest that computer software make documentation easier and patients prefer computerised documentation (Blaney, 2012; Waithera et al., 2017; Akhu-Zaheya et al., 2018). This suggests the necessity for a move from the paper-based system to electronic medical record system. Nurses must be trained on the system extensively to limit resistance to its adoption.

Age of respondents (21-30 years) was significantly associated with the level of practice of nursing documentation. Again, younger nurses with their youthful vigour from nursing school tend to stick with documentation values from school and this may account for their practice of nursing documentation. Also, younger nurses are typically subordinates and they are often left with the duty to document nursing procedures. This may account for their higher level of practice. Nurses in the surgical wards, paediatric and obstetric wards practiced documentation. These wards are rife with nursing procedures that require documentation compared with other wards. Also, the practice of nursing documentation was associated with the level of knowledge of nursing documentation. Andualem et al., (2019) reported a 
similar finding in a study conducted in Ethiopia. People with more knowledge on documentation tend to possess better attitudes Andualem et al., (2019) and practice documentation more often.

The study also identified some organizational factors that impacted positively on documentation practices such as the availability of operational standards for nursing care, in-service training on documentation and availability of documentation sheets. Hindrance factors mentioned by participants included lack of adequate staff, no obligation from the facility and lack of motivation from supervisors. Other factors identified included lack of time and nurses' apathy towards documentation. Similar findings were reported by a study undertaken among 286 registered nurses and midwives in the same facility and factors that hindered nursing documentation were mentioned to include the absence of nursing process policy, lack of further nursing process training and lack of nursing process clinical skills (Wahab, 2017). In Accra, Ghana, another cross-sectional study reported factors that influenced their inability to practice nursing documentation to include inadequate practical knowledge, work-overload and management's inability to provide the needed materials and time constraints (Clark, 2017). In Iran, the findings of a study showed that working problems and lack of motivation affected nursing records, lack of knowledge about the principles and standards of nursing were factors increasing poor nursing documentation (Bijani et al., 2016). Health institutions must endeavour to provide documentation training regularly to improve nurse knowledge and awareness whilst ensuring better access to documentation supplies.

\section{Study Limitations}

This study has provided evidence on the knowledge, attitudes and practices of nurses on nursing documentation at TTH. However, there are some limitations that must be considered. We did a survey among nurses and so we could not explore their experiences on documentation. Future qualitative studies involving nurses, midwives and nursing managers should be carried out. Also, this study may be prone to social desirability bias because the questionnaire was self-administered.

\section{Conclusion}

The level of knowledge of the majority of the nurses on nursing documentation was found to be adequate and the main source of knowledge of the participants was nursing schools. Also, participants had positive attitudes towards nursing documentation, whilst the practice of documentation was also noted to be frequent for majority of participants and more likely to be adequate. To strengthen nursing documentation, it is recommended that operational factors militating against nursing documentation be addressed by nursing managers and policy makers; such as equipping the Tamale Teaching Hospital with nursing staff, logistics and guidelines on nursing documentation.

\section{Authors' Contributions}

All authors made significant contributions towards the successful completion of this publication.

\section{Acknowledgement}

The authors are grateful to the Dean, Dr. Vida Yakong and all lecturers of the School of Nursing and Midwifery, University for Development Studies for their continuous training and support. Also, we fully acknowledge and are grateful for the time and effort spent by nurses and midwives at the TTH. We also express our appreciation to the Staff of the Research Unit of the TTH for both the administrative and ethical approvals given to us before the commencement of the study.

\section{References}

Adobasom-Anane, A. G. (2018). Retrospective Study of Snakebite Cases at The Tamale Teaching Hospital, Univeristy for Development Studies. Univeristy for Development Studies.

Akhu-Zaheya, L., Al-Maaitah, R., \& Bany Hani, S. (2018). Quality of nursing documentation: 
Paper-based health records versus electronicbased health records. Journal of Clinical Nursing, 27(3-4).

Allen, D. (1998). Record-keeping and routine nursing practice: the view from the wards. $J$ Adv Nur, 27(6), 1223-30.

Andualem, A., Asmamaw, T., Sintayehu, M., Liknaw, T., Edmealem, A., Bewuket, B., \& Gedfaw, M. (2019). Knowledge, attitude , practice and associated factors towards nursing care documentation among nurses in West Gojjam Zone public hospitals ,. Clin J Nurs Care Pract., 3, 1-13.

Arko, J. (2017). Code of Professional ConductNursing and Midwifery Council of Ghana, Nursing in Ghana. NursinginGhana.

Asamani, J. A., Amenorpe, F. D., Babanawo, F., Maria, A., \& Ofei, A. (2015). of inpatient care in eastern Ghana. British Journal of Nursing, 23(1), 48-54.

Bijani, M., Sadeghzadeh, M., A, K. J., \& Hannan, K. S. (2016). Factors influencing poor nursing documentation from the perspective of nursing staff. International Journal of Medical Research \& Health Sciences, 5(11), 717-718.

Bjorvell, C. (2002). Nursing Documentation in Clinical Practice: Instrument Development and Evaluation of a Comprehensive Intervention Programme. Karolinska Institutet, Stockholm, Sweden.

Blaney, C. D. (2012). The Influence of Changing Nurse Documentation Practices Have on Patient Satisfaction. University of Phoenix.

Broderick, M., \& Coffey, A. (2013). Personcentred care in nursing documentation. International Journal of Older People Nursing, 8 4, 309-318.

Buunaaisie, C., Iddrisu, O. A., Letitia, C., Abass, Y., Kyilleh, J., \& Abdul-Malik, A. (2018).
Project Report on Assessment of Nursing Documentation Practices in Five Hospitals in Tamale Metropolis: A Retrospective Records Review. 1-36.

Clark, E. K. (2017). Application of Nursing Process in Paediatric Care and The Factors Associated with Its Implementation at KorleBu Teaching Hospital and Princess Marie Louis Hospital in Accra. University of Cape Coast, Ghana.

Deriba, B. K., Sinke, S. O., Ereso, B. M., \& Badacho, A. S. (2017). Health professionals, job satisfaction and associated factors at public health centers in West Ethiopia. 1-7. https://doi.org/10.1186/s12960-017-0206-3

Hameed, Y. R., \& Allo, R. R. (2014). Assessment of Nurses' Knowledge About Nursing Documentation. Journal of Kufa for Nursing Science, 4(1).

Hassan, N. A., Shalzy, M. M., \& Aly, E. R. I. (2018). Assessing Nurses' Knowledge and Auditing their Practices Regarding Nursing Care Documentation. Port Said Scientific Journal of Nursing, 5(1), 95-112.

Hussainat Taiye, B. (2015). Knowledge and Practice of Documentation among Nurses in. IOSR Journal of Nursing and Health Science Ver. I, 4(6), 2320-1940.

Johnson, B. B. (2011). Nursing documentation as a communication tool: a case study from Ghana. Tromso University, Norway.

Kamil, H., Rachmah, R., \& Wardani, E. (2018b). What is the problem with nursing documentation? Perspective of Indonesian nurses. International Journal of Africa Nursing Sciences, 9(September), 111-114.

Karkkainen, O., Bondas, T., \& Eriksson, K. (2005). Documentation of individualized patient care: a qualitative meta synthesis. Nurs. Ethics, 12(2), 123-132. 
Keenan, G. M., Yakel, E., Tschannen, D., \& Mandeville, M. (2008). Documentation and the Nurse Care Planning Process. In Patient Safety and Quality: An Evidence-Based Handbook for Nurses (R. G. Hugh). Agency for Healthcare Research and Quality.

Machudo, S. Y., \& Mohidin, S. (2015). Nursing Documentation Project at Teaching Hospital in KSA. Int J Res Sci., 1(1), 1-7.

Mathioudakis, A., Rousalova, I., Gagnat, A. A., Saad, N., \& Hardavella, G. (2016). How to keep good clinical records. Breathe, 12(4), 371-375.

Mbabazi, P., \& Cassimjee, R. (2006). The quality of nursing documentation in a hospital in Rwanda. 8, 31-42.

McBride, S., Delaney, J. M., \& Tietze, M. (2012). Health information technology and nursing. Am J Nurs., 112(8), 36-42.

Mutshatshi, T. E., Mothiba, T. M., Mamogobo, P. M., \& Mbombi, M. O. (2018b). Recordkeeping: Challenges experienced by nurses in selected public hospitals. Curationis, 41(1).

Nakate, G., Dahl, D., Drake, K., \& Petrucka, P. (2015). Knowledge and Attitudes of Select Ugandan Nurses towards Documentation of Patient Care. African Journal of Nursing and Midwifery, 2(1), 056.

Nakate, G. M., Dahl, D., Petrucka, P., B. Drake, K., \& Dunlap, R. (2015). The Nursing Documentation Dilemma in Uganda: Neglected but Necessary. A Case Study at Mulago National Referral Hospital. Open Journal of Nursing, 05(12), 1063-1071.

Okaisu, E. M., Kalikwani, F., Wanyana, G., \& Coetzee, M. (2014). Improving the quality of nursing documentation: An action research project. Curationis, 37(1).

Okine, J. (2017). Perspectives of Nurses on
Continuity of Patient Care: A Study at The Trauma and Specialist Hospital, University of Ghana. University of Ghana.

Okine, Josephine. (2017). School of Nursing and Midwifery College of Health Sciences University of Ghana Perspectives of Nurses on Continuity of Patient Care : a Study At the Trauma and Specialist Hospital, Winneba By This Dissertation Is Submitted To the University of Ghana Lego. 10269473.

Oyebode, F. (2013). Clinical errors and medical negligence. Medical Principles and Practice, 22(4), 323-333.

Priest, C., Kooken, W. C., Ealey, K. L., Holmes, S. R., \& Hufeld, P. (2007). Improving baccalaureate nursing students understanding of fundamental legal issues through interdisciplinary collaboration. J. Nurs. Law, 11(1), 35-42.

Taiye, B. H. (2015). Knowledge and Practice of Documentation among Nurses in Ahmadu Bello University Teaching Hospital ( Abuth ) Zaria , Kaduna State . IOSR Journal of Nursing and Health Science, 4(6), 1-6.

Tasew, H., Mariye, T., \& Teklay, G. (2019). Nursing documentation practice and associated factors among nurses in public hospitals, Tigray, Ethiopia. BMC Research Notes, 12(1), 1-6.

Urquhart, C., Currell, R., Grant, M. J., \& Hardiker, N. R. (2018). Nursing record systems: Effects on nursing practice and healthcare outcomes. Cochrane Database of Systematic Reviews, 2018(5).

Wahab, O. (2017). Utilisation of The Nursing Process for Patient Care in Ghana: The Case of Nurses of Tamale Teaching Hospital. University of Cape Coast.

Yamane, T. (1967). Statistics: An Introductory Analysis (2nd ed.). Harper and Row. 